\section{SAT0453 ASSOCIATION OF COMORBID PULMONARY CONDITIONS WITH PATIENT-REPORTED OUTCOMES IN SYSTEMIC LUPUS ERYTHEMATOSUS}

P. Katz ${ }^{1}$, S. Pedro ${ }^{2}$, E. Alemao ${ }^{3}$, J. Mukherjee ${ }^{3}$, K. Michaud ${ }^{2} .{ }^{1}$ University of California, San Francisco; ${ }^{2}$ National Data Bank for Rheumatic Diseases, Wichita; ${ }^{3}$ Bristol-Myers Squibb, Princeton, USA

Background: Risk of chronic obstructive pulmonary disease (COPD) and allergic conditions, including asthma (AM), is elevated among SLE patients. ${ }^{1}$ Both AM and COPD negatively affect quality of life measured through patient-reported outcomes (PROs). Little research has examined the impact of AM and COPD on PROs in SLE, independent of SLE disease status.

Objectives: Determine the impact of AM/COPD on PROs in SLE, concurrently and longitudinally.

Methods: Data from 2 large, longitudinal, observational cohorts were examined (Lupus Outcomes Study, LOS: $n=796$; National Data Bank for Rheumatic Diseases, Forward: $n=2804$ ). AM and COPD were determined at study entry by selfreport. PROs included validated scales or items measuring physical functioning, fatigue, pain, cognitive function, depressive symptoms and global severity, although the cohorts included different PROs (Table). Multiple regression analyses examined differences between subjects with and without AM/COPD crosssectionally, controlling for age, sex, race, lupus duration, education, income, obesity, smoking, other comorbid conditions, and presence or history of renal involvement, clotting disorder or seizures. Longitudinal analyses examined PROs at 3 years (yrs) of follow-up, controlling for covariates above as well as baseline PRO values.

Results: LOS cohort was $92 \%$ female, mean age 47 years, $70 \%$ white, $42 \%$ ever smokers, mean lupus duration 13 years. Forward cohort was $94 \%$ female, mean age 51 years, $87 \%$ white, $38 \%$ ever smokers, mean lupus duration 16 years. $36 \%$ of LOS and $30 \%$ of Forward reported AM/COPD at study entry, compared to COPD prevalence of $7 \%{ }^{2}$ and AM prevalence of $9.7 \%{ }^{3}$ among US women. In cross-sectional analyses (Table), AM/COPD were associated with significantly worse scores on all PRO measures, except depressive symptoms (LOS). Longitudinal results were similar: AM/COPD were associated with worse scores on all PROs except SF-36 PF and Fatigue in LOS.

\begin{tabular}{lccc} 
Table. Multivariate Regression Analyses & \\
\hline & PRO variables & $\begin{array}{c}\text { Cross- } \\
\text { sectional }\end{array}$ & Longitudinal \\
\hline LOS & SF-36 Physical Function $(\mathrm{PF})^{\dagger}$ & $-9.2(<0.0001)^{*}$ & $-1.8(0.26)$ \\
& SF-36 Fatigue & $6.6(<0.0001)$ & $2.4(0.09)$ \\
& CESD & $1.5(0.10)$ & $1.7(0.04)$ \\
Forward & MOS Cognitive & $-6.6(<0.0001)$ & $-3.3(0.01)$ \\
& HAQ II & $0.2(<0.0001)$ & $0.2(<0.0001)$ \\
& Fatigue $(0-10)$ & $0.7(<0.0001$ & $0.8(<0.0001)$ \\
& PHQ8 & $2.0(0.01)$ & $1.9(0.006)$ \\
& Pain $(0-10)$ & $0.8(<0.0001)$ & $0.8(<0.0001)$ \\
& Trouble thinking or & $1.5(1.2-1.8)$ & $1.2(1.0-1.5)$ \\
& remembering & & \\
& Global severity (0-10) & $0.5(<0.0001)$ & $0.6(<0.0001)$ \\
\hline * & &
\end{tabular}

*Beta ( $p$ value) from multiple linear regression analyses, except Forward 'trouble thinking or remembering', which is odds ratio $(95 \% \mathrm{Cl})$

'Lower scores=worse status. Otherwise, higher scores=worse status

CESD=Centre for Epidemiologic Studies Depression scale; $\mathrm{HAQ}=$ Health Assessment

Questionnaire; PHQ8=Patient Health Questionnaire depression inventory

Conclusions: AM/COPD are more common in SLE than the general population and are independently associated with worse outcomes on a wide range of PROs, even after controlling for sociodemographic and lupus characteristics. Findings suggest that physicians should screen for pulmonary comorbidities and ensure adequate treatment for these conditions. Future analyses of PROs in SLE should include AM/COPD as important comorbid conditions.

\section{REFERENCES:}

[1] Shen T-C, et al. PLoS One 2014;9:e91821.

[2] Ford ES, et al. Chest 2013;144:284-305.

[3] www.cdc.gov/asthma/most_recent_data.htm. Last accessed 4 January 2018.

Disclosure of Interest: P. Katz Grant/research support from: Bristol-Myers Squibb, S. Pedro Grant/research support from: Bristol-Myers Squibb, E. Alemao Shareholder of: Bristol-Myers Squibb, Employee of: Bristol-Myers Squibb, J. Mukherjee Employee of: Bristol-Myers Squibb, K. Michaud Grant/research support from: Pfizer and Rheumatology Research Foundation, Employee of: University of Nebraska Medical Centre and National Data Bank for Rheumatic Diseases DOI: 10.1136/annrheumdis-2018-eular.1795

\section{SAT0454 \\ IN PRIMARY SJÖGREN'S SYNDROME BLOOD CD180- NEGATIVE B CELLS ARE INCREASED IN PATIENTS WITH HIGH SYSTEMIC DISEASE ACTIVITY}

R. Felten ${ }^{1}$, C. Mayeur-Rousse ${ }^{2}$, L. Miguet ${ }^{2}$, L. Arnaud ${ }^{1}$, E. Chatelus ${ }^{1}$, R.-

M. Javier ${ }^{1}$, H. Petit ${ }^{1}$, J. Sibilia' ${ }^{1}$, C. Sordet ${ }^{1}$, L. Fornecker ${ }^{2}$, L. Mauvieux ${ }^{2}$, J.-

E. Gottenberg ${ }^{1} .{ }^{1}$ Rheumatology, ${ }^{2}$ Hematology, Hautepierre, Strasbourg, France

Background: CD180, a related member of the Toll-like receptor family, seems to be underexpressed at the plasma membrane in circulating B cells of auto-immune disease (systemic lupus especially), ${ }^{1}$ and is lost in various B-cell lymphomas except marginal zone lymphomas (MZL), the most severe complication of primary Sjögren's Syndrome (pSS). ${ }^{2}$

Objectives: To define the expression of CD180 at the plasma membrane of circulating B cells of pSS and determine its association with systemic disease activity. Methods: The expression of CD180 at the plasma membrane of circulating B cells was studied by flow cytometry, in 69 successive patients with pSS, in comparison to patients with systemic lupus (SLE) $(n=7)$ and healthy controls $(n=28)$.

Results: The cohort of pSS was composed of 65 women for 4 men, median age of 59 years. ${ }^{25-89}$ Median clinESSDAI was 2 [0-18], 46 patients with a clinESS$\mathrm{DAI}>0$ (low disease activity), 18 patients with a clinESSDAI $>5$ (moderate disease activity) and 6 patients $\geq 14$ (high disease activity). The more frequently involved domains of the ESSDAI were biological $(39 \%, n=27)$, glandular $(32 \%, n=22)$, articular $(20 \%, n=14)$, and peripheral neurological $(9 \%, n=6)$ domains. Fourty patients were anti-SSA+, 16 anti-SSA and anti-SSB+. 18 had a hypergammaglobulinemia 17 a hypocomplementemia, 7 a cryoglobulinemia, 18 a rheumatoid factor and 9 a lymphopenia.

\section{CD180-negative B cells in pSS, SLE and controls}

The proportion of total lymphocytes, B cells, T cells, NK cells or CD180-negative $B$ cells $(0.85 \%$ vs $0.95 \%, p=0.64)$ was not significantly different between $p S S$ patients and healthy controls.

Patients with SLE had significantly fewer lymphocytes ( 860 vs $1544, p=0.0012$ ), B cells and T cells than those with pSS ( $p=0.023$ and $p=0.0016) ; C D 180$-negative $B$ cells tended have a higher proportion in SLE than in pSS patients $(1.9 \%$ vs $0.85 \%, p=0.055$ ).

- Increase of CD180-negative B cells in patients with pSS and high systemic disease activity

CD180-negative B cells subset was significantly higher in patients with high systemic disease activity $(2.55 \%$ CD180-negative B cells in patients with clinESSDAI $\geq 14$ vs $0.8 \%$ in patients with clinESSDAI $<14, p=0.036)$. CD180-neg ative $B$ cells subset was also significantly higher in patients with anti-SSA and anti-SSB+ $(1.9 \%$ vs $0.8 \%, \mathrm{p}=0.0082)$ and in patients with markers of B-cell activation: hypergammaglobulinemia $(1.7 \%$ vs $0.75 \%, p=0.034)$, hypocomplementemia ( $2.1 \%$ vs $0.8 \%, p=0.0021$ ), cryoglobulinemia ( $4.6 \%$ vs $0.8 \%, p=0.0097$ ), presence of rheumatoid factor $(1.7 \%$ vs $0.7 \%, p=0.015)$

Conclusions: Blood CD180-negative B cells are increased in pSS patients with high systemic disease activity or in immunologically active pSS. Fonctionnality of CD180 and the prognostic interest of CD180 staining on blood B cells to predict lymphoma in pSS patients, have now to be studied.

\section{REFERENCE:}

[1] Kikuchi Y, et al. RP105-negative B cells in patients with Sjögren's syndrome.

[2] Mayeur-Rousse C, et al. CD180 expression in B-cell lymphomas: A multicenter GEIL study.

Disclosure of Interest: None declared DOI: 10.1136/annrheumdis-2018-eular.4669

\section{SAT0455 CLINICAL AND IMMUNOLOGICAL CHARACTERISTICS OF PATIENTS WITH SUSPECTED SJÖGREN SYNDROME AND ANTI-RO52 POSITIVE ANTIBODIES}

R. Solans-Laque $^{1}$, J. Loureiro ${ }^{1}$, A. Marin ${ }^{2}$, F. Martinez-Valle ${ }^{1}$, M. Alberola ${ }^{3}, \mathrm{~J}$ J. Puig ${ }^{4}$, J. Mestre ${ }^{1}$, S. Bujan'. ${ }^{1}$ Autoimmune Diseases Unit. Internal MedicineDepartment, ${ }^{2}$ Immunology; ${ }^{3}$ Pathology, ${ }^{4}$ Opthalmology, Vall Hebron University Hospital, Barcelona, Spain

Background: Anti-Ro52 antibodies have been described in patients with Sjögren syndrome (SS) but there are limited data on their significance, and they are not included in the diagnostic criteria of SS.

Objectives: To analyse the clinical and immunological characteristics of patients with suspected SS and positive anti-Ro52 antibodies, and to assess the diagnos tic value of this antibodies

Methods: retrospective study of all patients evaluated at our Department between January 2000 and January 2016 for possible SS in which anti-Ro52 abs. were determined. Patients were classified as having SS, according to the 2002 